\title{
1 The evolution of sex differences in disease genetics
}

William P. Gilks ${ }^{\dagger \S}$, Jessica K. Abbott ${ }^{* \S}$, Edward H. Morrow ${ }^{\dagger}$

${ }^{\dagger}$ Evolution, Behaviour and Environment group, School of Life Sciences, University of Sussex, Falmer BN1 9QG, United Kingdom, URL http://www.sussex.ac.uk/lifesci/morrowlab/

7 *Experimental Evolution, Ecology \& Behaviour group, Department of Biology, Section for Evolutionary Ecology, Lund University,

8 Sölvegatan 37, 22362 Lund, Sweden, URLs http://jessicakabbott.com/ and http://www4.lu.se/o.o.i.s/25872

$9 \S$ These authors contributed equally.

$14^{\text {th }}$ November 2013

\section{Abstract}

There are significant differences in the biology of males and females, ranging from biochemical pathways to behavioural responses, which are relevant to modern medicine. Broad-sense heritability estimates differ between the sexes for many common medical disorders, indicating that genetic architecture can be sexdependent. Recent genome-wide association studies (GWAS) have successfully identified sex-specific and sex-biased effects, where in addition to sex-specific effects on gene expression, twenty-two medical traits have sex-specific or sex-biased loci. Sex-specific genetic architecture of complex traits is also extensively documented in model organisms using genome-wide linkage or association mapping, and in gene disruption studies. The evolutionary origins of sex-specific genetic architecture and sexual dimorphism lie in the fact that males and females share most of their genetic variation yet experience different selection pressures. At the extreme is sexual antagonism, where selection on an allele acts in opposite directions between the sexes. Sexual antagonism has been repeatedly identified via a number of experimental methods in a range of different taxa. Although the molecular basis remains to be identified, mathematical models predict the maintenance of deleterious variants that experience selection in a sexdependent manner. There are multiple mechanisms by which sexual antagonism and alleles under sexdifferential selection could contribute toward the genetics of common, complex disorders. The evidence we review clearly indicates that further research into sex-dependent selection and the sex-specific genetic architecture of diseases would be rewarding. This would be aided by studies of laboratory and wild animal populations, and by modelling sex-specific effects in genome-wide association data with joint, gene-bysex interaction tests. We predict that even sexually monomorphic diseases may harbour cryptic sexspecific genetic architecture. Furthermore, empirical evidence suggests that investigating sex-dependent epistasis may be especially rewarding. Finally, the prevalent nature of sex-specific genetic architecture in disease offers scope for the development of more effective, sex-specific therapies.

\section{Funding}

38 This work was supported by the European Research Council (WPG and EHM; Starting Grant \#280632), a

39 Royal Society University Research Fellowship (EHM), the Swedish Research Council (JKA), and the

40 Volkswagen Foundation (JKA). The funders had no role in study design, data collection and analysis, 41 decision to publish, or preparation of the manuscript. 


\section{Competing interests}

44 The authors declare that they have no competing financial interests.

45

46

47

48

49

50

51

52

53

54

55

56

57

58

59

60

61

62

63

64

65

66

67

\section{Introduction}

Sex, and the existence of two sexes, has revolutionised life on Earth. The success of sexual reproduction is attributed to recombination between parental chromosomes, which accelerates the loss of deleterious alleles and the proliferation of advantageous ones [1,2]. The difference in gamete size between males and females is a fundamental property of almost all sexual species. Yet sexual dimorphism extends far beyond this, from cellular and anatomical specialisation to secondary sexual traits such as ornamentation and behaviour. Furthermore, there are differences in gene co-expression and metabolome networks between the sexes [3-5]. It is therefore not surprising that in the field of medicine, males and females frequently differ in core features of disease [6].

The genetic basis of disease has been intensely researched, with the aim of providing improved diagnosis and therapy. Heritable diseases can be classified as being rare with monogenic aetiology (caused by a single mutation), or common (prevalence $0.1-1 \%$ ), caused by multiple genetic variants, each with high population frequency but small individual contribution to disease risk [7,8]. For these genetically complex diseases and traits, genome-wide association studies (GWAS) have been successful at identifying loci, but the heritability accounted for by main effects, and by polygenic risk score, remains conspicuously low $[9,10]$. This deficit is stimulating the consideration of other factors such as the environment and epistasis [11]. Sex differences in the genetic architecture of common diseases have been known for some time [12], and recent analysis of large GWAS datasets has resulted in an unprecedented rise in the number of known sex-specific loci for human diseases and quantitative traits (see next section). Whilst this fact alone should encourage further investigation, evolutionary theory also predicts the existence of sexspecific genetic architecture for complex traits via sex-specific or sexually antagonistic selection.

Males and females share genes and genetic variation (excepting the Y chromosome), yet often have divergent optimum conditions for survival and reproduction [13]. A recent model predicts that even a marginal difference in selection pressure rapidly amplifies the contribution deleterious alleles make to trait architecture [14]. Additionally, opposing selection pressures on a shared trait creates sexual antagonism, in which the strength of positive selection for an allele in one sex will allow it to be maintained even if it is deleterious to the other sex (see section 3 ) $[15,16]$. In this review we summarise recent evidence for the sex-specific genetic architecture of common diseases, and the evidence for sexual antagonism in the light of evolutionary theory. We also propose new mechanisms by which sexual antagonism can contribute towards the genetic architecture of disease, and guidelines for the identification of sex-specific genetic effects. 


\section{Evidence for sex-specific genetic architecture}

83 Broad-sense heritability is the proportion of phenotypic variance in a population sample that can be 84 attributed to genetic variation [17]. With identical genetic architecture, and assuming a common environment, trait heritability should be equal in male and female samples. However, in a study of twenty quantitative traits in humans, eleven showed significant sex-bias in heritability [18]. Following a PubMed literature search, we identified eighteen independent studies in humans (representing thirty-one traits) that provided separate heritability estimates for males and females, and also stated whether the difference was statistically significant. A summary of these data is presented in Figure 1, showing that while fifteen traits did not exhibit significant sex-bias in heritability, thirteen had a higher heritability in females, and three a higher heritability in males. Although there may be some bias in these studies (non-reporting, nonindependence of traits or prior selection of traits with known sexual dimorphism), they illustrate that the heritability of complex traits is commonly sex-biased across a range of phenotype classes.

It is well known that non-genetic factors influence differences in heritability, the most obvious being sex hormones (androgens, oestrogens and progesterones, secreted from the gonads). These can create systemic differences between males and females for trait expression, which in turn affects disease risk and heritability, for example the protective effect of oestrogen on heart disease [19]. However, experiments using hormone treatment and gonadectomy show that some gender differences in phenotypes, such as immune response, behaviour, and toxin resistance, are not determined by sex hormones but by sex chromosome dosage [20-22]. This implies that heritability differences are not always caused by sex hormones, and can be caused by sex-specific differences in genetic architecture, whereby a genetic variant has a different phenotypic outcome depending on whether it is expressed in a male or female environment.

The molecular genetic evidence for sex-specific genetic architecture is strong. For gene expression in human cell lines, $15 \%$ of SNPs that control gene expression (expression quantitative trait loci or eQTL) do so in a sex-specific manner, even in the absence of sex hormones [23]. For complex traits, GWAS have identified many robust sex-specific loci across a range of human phenotypes. These results are summarised Table 1, which shows thirty-two loci with sex-dependent effects in the twenty-two

108 traits studied. The majority of the effects were sex-specific (twenty-eight loci significant in one sex only) although five sex-biased effects were also reported (significant in both sexes but different magnitude of 110 effect). One opposite effect direction locus has also been reported from a GWAS (for recombination rate

111 [24]). Model organisms such as laboratory mice Mus musculus and the fruit fly Drosophila melanogaster 112 have been used successfully in many phenotype-mapping projects, facilitated by the controlled 113 environment and flexibility of experimental design. Indeed, many sex-specific eQTL have been identified 114 in the fruit fly D. melanogaster [25] and laboratory mice [26]. For genetically complex traits, sex-specific 115 loci have been identified for sleep patterns and ageing in D. melanogaster, and for fat mass and skeletal 116 traits in mice [27-30].

117 Gene manipulation studies of model organisms have identified hundreds of genes with sexually 118 dimorphic effects on disease-related phenotypes. Interestingly, not only have sex-specific and sex-biased 119 effects been observed, but also sex-reversed and sexually pleiotropic effects (when the same locus affects 120 different traits in males and females; see section 5). For example, murine vitamin D receptor disruption 
121 causes weight loss in males but decreased bone density in females [31], and p53 over-expression in $D$. 122 melanogaster increases male life-span but reduces that of females [32]. Furthermore, a screen of 1,332 D. 123 melanogaster P-element insertion lines identified forty-one mutations that had sexually dimorphic effects 124 on life-span including six that were in opposite directions [33]. Although gene disruption studies do not 125 precisely reproduce the effect of natural genetic variation, they demonstrate that different pathways can 126 control the same trait. The question then is: how does this sexual dimorphism in genetic effects arise? 127 Insights from evolutionary biology are of great value here, since theory about the origin and evolution of 128 sex differences is well-developed, both on the phenotypic and on the genetic level.

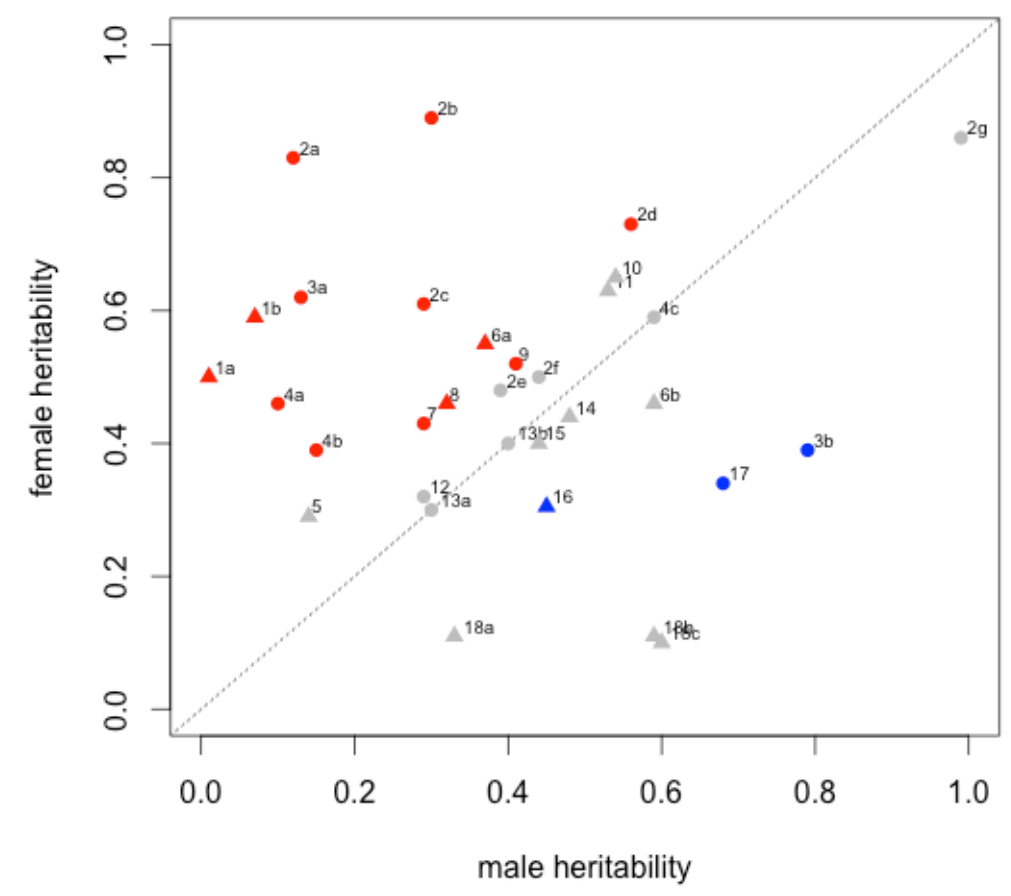

Figure 1: Comparison of male vs female narrow-sense heritability estimates from human studies. Red and blue131 coloured data points indicate significant differences between the sexes. Grey data points indictate no significant 132 difference between the sexes. Triangles indicate a binary/qualitative phenotype. Circles indicate a 133 continuous/quantitative phenotype. 1a: Drive for thinness. 1b Body Dissatisfaction [103]. 2a: Waist diameter. 2b: 134 Waist-height ratio. 2c: Body-mass index. 2d: Peripheral body fat. 2e: Hip diameter. 2f: Body weight. 2g: Body height 135 [104]. 3a: Triglyceride serum level. 3b: LDL cholesterol serum level [105]. 4a: Lung FEV1 (forced exit volume). 4b: 136 Lung $\mathrm{D}_{\text {LCO }}$ (diffusing capacity). 4c: Lung VC (vital capacity) [106]. 5: Geriatric depression [107]. 6a: Smoking 137 initiation. 6b: Regular tobacco use [108]. 7: Sleep reactivity (insomnia) [109]. 8: Alcohol dependence [110]. 9: 138 Subjective well-being [111]. 10: Reading disability [112]. 11: Reading difficulties [113]. 12: Self-esteem [114]. 13a: 139 Respiratory sinus arythmia. 13b: Heart beat entropy [115]. 14: Tension-type headache [116]. 15: Lower back pain 140 [117]. 16: Seasonal mood change [118]. 17: Protein C sensitivity [119]. 18a: Drug use. 18b: Tobacco use. 18c: 141 Alcohol use [120]. 
Table 1: Loci with sex-dependent effects on human phenotypes, identified through genome-wide SNP or CNV analysis

\begin{tabular}{|c|c|c|c|c|c|c|c|c|c|}
\hline Phenotype & Sex test & $\begin{array}{c}\text { Individuals } \\
\text { tested }\end{array}$ & Gene & Chromosome band & SNP & MAF & Male effect ${ }^{\dagger}$ & Female effect ${ }^{\dagger}$ & Reference \\
\hline Mitochondrial DNA levels & Separate & 384 & "MRPL37 & 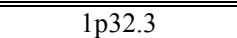 & rs 10888838 & 0.11 & 0.81 & 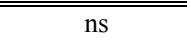 & [121] \\
\hline Heart beat rate (QT interval) & Separate & 3761 & NOS1AP & $1 \mathrm{q} 23.3$ & rs10494366 & 0.29 & 3.08 & 2.09 & [122] \\
\hline Waist-height ratio & Sep \& p-diff & 175585 & LYPLAL1/SLC30A10 & $1 \mathrm{q} 41$ & rs 4846567 & 0.29 & $\mathrm{~ns}$ & 0.06 & [123] \\
\hline Waist-height ratio & Separate & 190803 & " & 1 & rs 2820443 & 0.29 & $\mathrm{~ns}$ & 0.05 & [124] \\
\hline Visceral adiposity & Separate & 117857 & THNSL2 & $2 \mathrm{p} 11.2$ & rs 1659258 & 0.35 & ns & Z-score 1.5 & [125] \\
\hline Mitochondrial DNA levels & Separate & 384 & RNF144 & $2 \mathrm{p} 25.1$ & rs 2140855 & 0.39 & ns & 0.32 & [121] \\
\hline Waist-height ratio & Sep \& p-diff & 175585 & GRB14/COBLL1 & $2 \mathrm{q} 24.3$ & rs 10195252 & 0.44 & ns & 0.05 & [123] \\
\hline “ & Separate & 190803 & “ & “ & rs6717858 & 0.44 & ns & 0.05 & [124] \\
\hline Plasma homocysteine & Separate & 1679 & CPS1 & $2 q 34$ & rs 1047891 & 0.30 & $\mathrm{~ns}$ & 0.04 & [126] \\
\hline Glycine levels & Separate & 3343 & “ & “ & rs715 & 0.24 & $\mathrm{~ns}$ & 0.23 & [3] \\
\hline Crohn's Disease & Separate & 8463 & ATG16L1 & $2 \mathrm{q} 37.1$ & rs3792106 & 0.40 & ns & OR 1.48 & [127] \\
\hline Waist-height ratio & Sep \& p-diff & 175585 & PPARG & 3p25.2 & rs 4684854 & 0.42 & $\mathrm{~ns}$ & 0.04 & [124] \\
\hline Waist-height ratio & Sep \& p-diff & 175585 & ADAMTS9 & $3 \mathrm{p} 14.1$ & rs6795735 & 0.19 & ns & 0.05 & [123] \\
\hline Recombination rate & Separate & 2500 & RNF212 & $4 \mathrm{p} 16.3$ & rs11939380 & 0.33 & $+118 \mathrm{cM}$ & ns & [128] \\
\hline “ & Separate & 19578 & “ & “ & rs 1670533 & 0.22 & $-69.4 \mathrm{cM}$ & $+88.2 \mathrm{cM}$ & {$[24]$} \\
\hline Uric acid concentration & p-diff & 28141 & SLC2A9 & $4 \mathrm{p} 16.1$ & rs 734553 & 0.26 & -0.22 & -0.40 & [129] \\
\hline Sex-hormone binding globulin & Separate & 21791 & UGT2B15 & $4 q 13.2$ & rs 293428 & 0.30 & -0.03 & ns & [130] \\
\hline Uric acid concentration & p-diff & 28141 & ABCG2 & $4 \mathrm{q} 22.1$ & rs 2231142 & 0.12 & 0.22 & 0.13 & [129] \\
\hline Waist circumference & Sep \& p-diff & 199499 & MAP3K1 & $5 \mathrm{q} 11.2$ & rs11743303 & 0.19 & ns & 0.03 & [124] \\
\hline Low-density lipoprotein (LDL) & Sep \& p-diff & 20512 & HMGCR & $5 \mathrm{q} 13.3$ & rs 12654264 & 0.38 & -4.03 & ns & [131] \\
\hline Thyroid stimulating hormone & Separate & 26420 & PDE8B & $5 \mathrm{q} 13.3$ & rs6885099 & 0.29 & -0.17 & -0.12 & [132] \\
\hline Naso-pharyngeal cancer & Separate & 1437 & MICA/HCP5 & $6 \mathrm{p} 21.33$ & na $(\mathrm{CNV})$ & 0.03 & OR 3-141 & ns & [133] \\
\hline Waist-height ratio & Sep \& p-diff & 175585 & VEGFA & $6 \mathrm{p} 21.1$ & rs6905288 & 0.45 & ns & 0.05 & [123] \\
\hline Thyroid stimulating hormone & Separate & 26420 & PDE10A & $6 \mathrm{q} 27$ & rs 753760 & 0.50 & 0.13 & 0.08 & [132] \\
\hline Pro-insulin levels & GWAMA* & 27079 & DDX31 & $9 \mathrm{q} 34.13$ & rs306549 & 0.24 & 0.04 & ns & [88] \\
\hline Obesity \& Osteoparosis (bivariate) & Separate & 4355 & SOX6 & $11 \mathrm{p} 15.1$ & rs 297325 & 0.20 & OR 1.75 & $\mathrm{~ns}$ & [134] \\
\hline Triglyceride levels & Sep \& p-diff & 24273 & APOA5/BUD13 & $11 \mathrm{q} 23.3$ & rs 28927680 & 0.07 & 0.13 & ns & [131] \\
\hline Type II Diabetes & Separate & 149000 & CCND2 & $12 \mathrm{p} 13.32$ & rs 11063069 & 0.21 & OR $1.08-1.16$ & ns & [135] \\
\hline Type I Diabetes ${ }^{*}$ & Separate & 27530 & CTSH & $15 \mathrm{q} 25.1$ & rs3825932 & 0.30 & OR $1.13-1.27$ & $\mathrm{~ns}$ & [136] \\
\hline Thyroxine levels (FT4) & Separate & 17498 & LPCAT2/CAPNS2 & $16 \mathrm{q} 12.2$ & rs6499766 & 0.48 & 0.02 & ns & [132] \\
\hline Thyroid stimulating hormone & Separate & 26420 & MAF & $16 \mathrm{q} 23.2$ & rs3813582 & 0.38 & 0.12 & 0.06 & [132] \\
\hline Recombination rate & Separate & 2500 & $17 \mathrm{q} 21.31$ region & $17 \mathrm{q} 21.31$ & rs 2668622 & 0.20 & ns & $+124 \mathrm{cM}$ & [128] \\
\hline Height & Separate & 1625 & NEDD4L & $18 \mathrm{q} 21.31$ & CNP12587 & 0.02 & $\mathrm{~ns}$ & $-8.1 \%$ & [137] \\
\hline Thyroxine levels (FT4) & Separate & 17146 & NETO1/FBXO15 & $18 \mathrm{q} 22.3$ & rs7240777 & 0.47 & ns & -0.08 & [132] \\
\hline Type II Diabetes & Separate & 149000 & GIPR & $19 \mathrm{q} 13.32$ & rs8108269 & 0.31 & ns & OR 1.06-1.14 & [135] \\
\hline High-density lipoprotein (HDL) & Sep \& p-diff & 11528 & PLTP & $20 \mathrm{q} 13.12$ & rs 7679 & 0.18 & ns & 1.68 & {$[131]$} \\
\hline
\end{tabular}

MAF Minor allele frequency. Value for similar HapMap population sample stated when study sample MAF not available

$\dagger$ Effect value is for the correlation coefficient $\beta$ unless otherwise stated. OR Odds ratio, $95 \%$ confidence intervals.

$¥$ Result of separate-sex analysis of SNPs previously identified in a standard, main-effects analysis.

* GWAMA 'Genome-wide analysis, meta-analysis' (Magi et al 2010)

SNP rs1047891 previously known as rs 7422339 . 


\section{Sexual antagonism}

150 Sexual antagonism is a genetic conflict resulting from sex-specific selection acting on a shared genome. A 151 subcategory of sexual antagonism is intra-locus sexual conflict, where a trait is controlled by the same 152 genes, and is distinct from inter-locus sexual conflict, which concerns reproductive interactions involving 153 different loci in each sex. Sexual antagonism has now been demonstrated in a wide variety of taxa, 154 including plants, birds, mammals, and insects [16,34]. Anisogamy (difference in gamete size) is 155 considered to be the ultimate source of sex-specific selection [35,36], although ecological factors can also 156 play a role in shaping patterns of sex-specific selection [37]. The fact that males produce many small 157 gametes and females few large gametes means that reproductive strategies are fundamentally different 158 across the sexes, which is thought to result in the evolution of sexual dimorphism [38]. However these 159 divergent phenotypes must be developed from a common pool of genetic information, making it difficult 160 to simultaneously achieve optimum trait values in both sexes. Thus, for certain traits a conflict will be 161 maintained and the sexes will be displaced from their optimum phenotypes. For example, when selection 162 on females was completely removed, experimentally evolved fruit flies became masculinized in a number 163 of traits demonstrating that males had previously been displaced from their phenotypic optimum by 164 counter-selection in females (reviewed in [39]). Pedigree analysis of wild animal populations has also 165 demonstrated a negative intersexual genetic correlation for fitness i.e. genotypes producing successful males produce unsuccessful females and vice versa $[40,41]$.

167 More formally, sexual antagonism occurs when genetically correlated traits have opposite effects 168 on male and female fitness. In the simplest case, increasing values of a single trait would increase fitness 169 in one sex and decrease it symmetrically in the other sex (Figure 2a). In this case the trait is assumed to be 170 positively correlated between the sexes. However more complicated patterns are also possible, such as 171 opposite fitness effects of different correlated traits (Figure 2b-c) or asymmetric patterns of selection 172 (Figure 2d). Consistent with this, a recent study demonstrated that human height was likely to be subject 173 to sexual antagonism: within sibling pairs, men of average height had higher fitness while shorter women 174 had higher fitness [42]. This means that the fitness effect of a given height-determining allele will be 175 context-dependent in terms of sex, and that the population as a whole will be unlikely to evolve towards a 176 shorter phenotype, despite directional selection in females, because of counter-selection in males. One of 177 the major evolutionary implications of sexual antagonism is the maintenance of genetic variation that is 178 deleterious to one sex. Although this has not been fully demonstrated at the molecular level, the 179 population dynamics of a synthetic sexually antagonistic allele in a laboratory D. melanogaster study 180 accurately follows predictions of mathematical models $[43,44]$. 


\section{Sex-specific fitnesses}

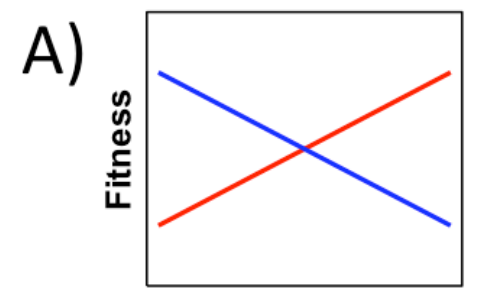

Trait 1

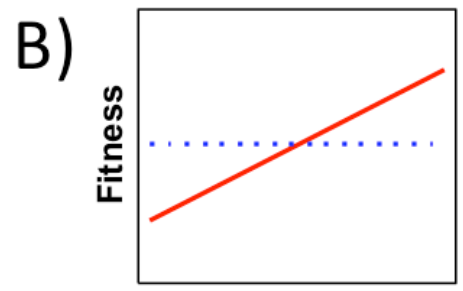

Female Trait 1

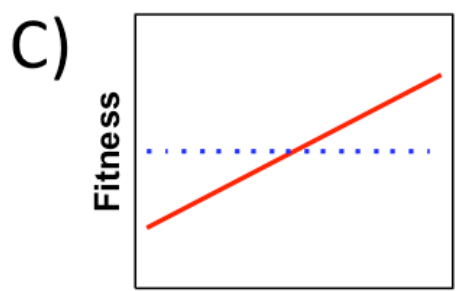

Female Trait 1

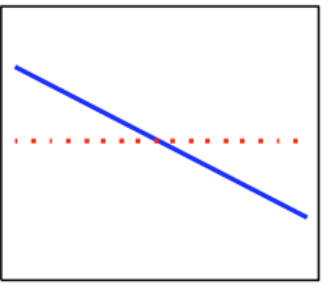

Male Trait 2

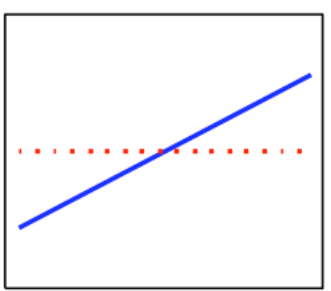

Male Trait 2

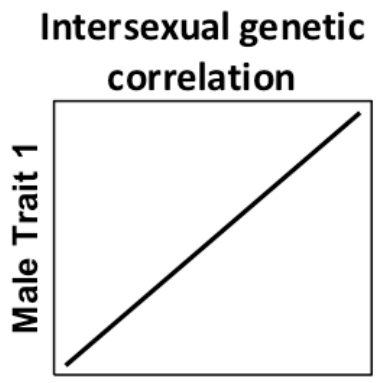

Female Trait 1

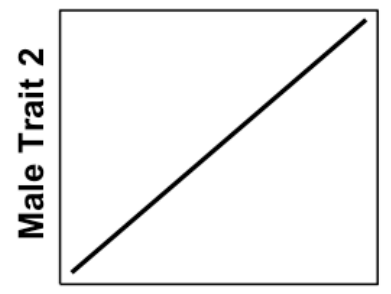

Female Trait 1

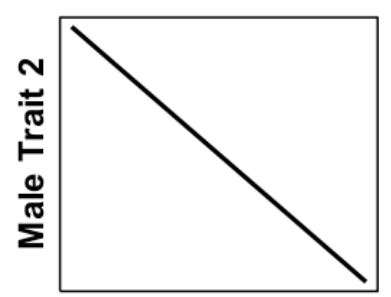

Female Trait 1

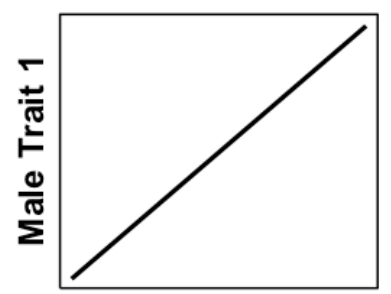

Female Trait 1

\section{D)}

Trait 1

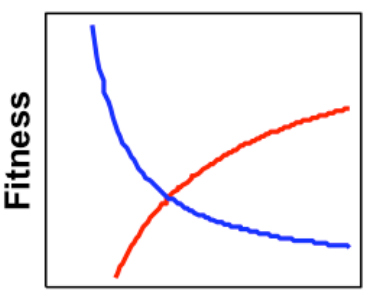

Figure 2: The various forms sexual antagonism can take. Female fitness functions are shown with red lines, male with blue lines, and the intersexual genetic correlation with black lines. A. The simplest case (also known as intralocus sexual conflict) is where the same trait has opposite and approximately symmetric fitness effects on males and females. The intersexual genetic correlation for the traits is high and positive. B. Sexual antagonism can also occur when different traits have a high positive intersexual genetic correlation, but are selected in opposite directions in males relative to females. In the unselected sex (broken lines), selection for the trait in question might be weakly positive, neutral, or even absent if the trait is sex-limited. C. Although no empirical examples of this type have yet been demonstrated, it is also possible that traits with a strong negative intersexual genetic correlation could be subject to sexual antagonism, assuming both traits are selected concordantly across the sexes. A negative intersexual genetic correlation could occur when the same gene product is incorporated in competing alternative pathways. D. It should also be pointed out that selection pressures need not be completely symmetric. Non-linear relationships are also possible. 


\section{Sexual dimorphism and resolution of sexual antagonism}

199 A sexually antagonistic trait is expected to go through several evolutionary stages (see Figure 3 for more

200 detail $[16,38])$.

201 1. Initially, the trait is monomorphic and under weak stabilizing selection.

202 2. A change in the physical or social environment causes the previously concordant trait to become subject

203 to opposite patterns of sex-specific directional selection. This is the most severe stage of antagonism.

204 3. Sexual dimorphism then evolves, causing the sexes to come closer to their respective phenotypic 205 optima, but the antagonism is only partially resolved.

206 4. Finally, the sexes reach their optimal phenotype values and the sexual antagonism is completely 207 resolved.

209 Most research to date has focused on sexually dimorphic traits, under the assumption that this dimorphism

210 is an indicator of sex-specific phenotypic optima. However the stage of the most severe antagonism and 211 the largest gender load $[45,46]$ is in fact before the trait in question becomes sexually dimorphic, and gene 212 expression data from D. melanogaster suggested that most sex-biased genes had already reached their 213 phenotypic optima [47]. In addition, if sexual antagonism results from correlated expression of different 214 traits across the sexes, monomorphism in a given trait may not be informative about its likelihood of being 215 subject to sexual antagonism [48]. This speaks in favour of casting a broad net when searching for 216 sexually antagonistic loci, and not only investigating traits that are already sexually dimorphic.

217 Proposed mechanisms for the resolution of sexual antagonism include the evolution of sex-linked 218 modifiers, alternative splicing, or gene duplication [38,49]. Gene duplication is a popular theory as to how 219 genes can escape sexual antagonism, by allowing each copy to evolve independently for each sex [50]. 220 Specifically, this would include genes that are activated by sex hormones or have sex-specific 221 methylation, and are thus expressed at different levels in each sex. There is debate about the time-scale of 222 the resolution of sexual antagonism [49,51-55], but regardless of whether the process is fast or slow in 223 evolutionary time, the outcome is always sex-specific genetic architecture. In this sense, sex-specific 224 genetic architecture in disease is likely to be an indirect result of past sex-specific or sexually antagonistic 225 selection. Sexual antagonism or sex-specificity could contribute towards disease risk directly, in which 226 case the sex-specific or sexually antagonistic alleles are themselves a risk factor [14], or indirectly, 227 because in its resolved state, sexual dimorphism creates its own intrinsic sex-specific risk factors, such as 228 behavioural and anatomical specialisation. 


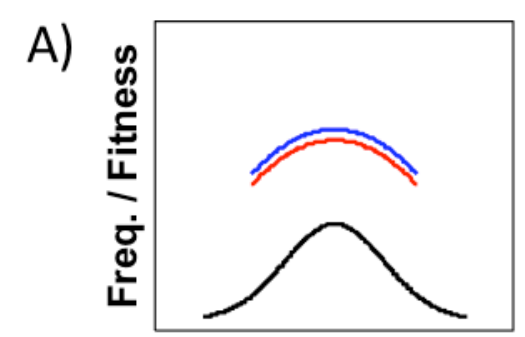

Trait 1

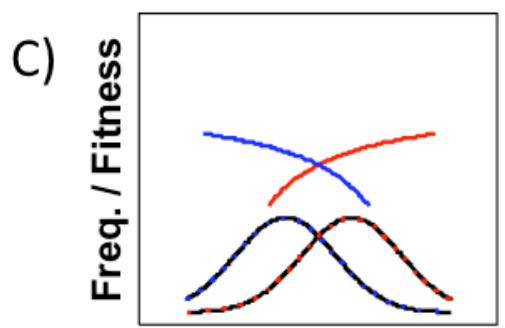

Trait 1

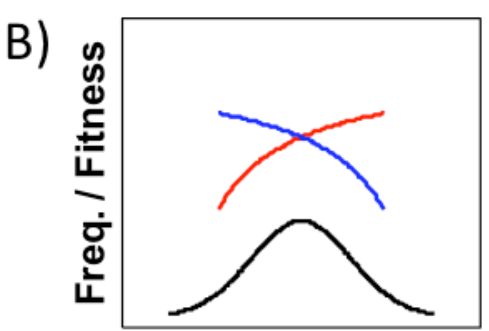

Trait 1

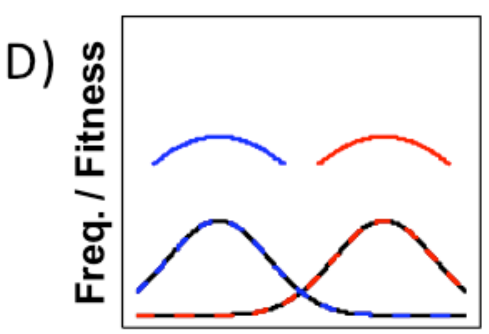

Trait 1

Figure 3: Predicted stages in the resolution of sexual antagonism. A. Initially, the trait is monomorphic and under weak stabilizing selection. B. A change in the physical or social environment causes the previously concordant trait to become subject to opposite patterns of sex-specific directional selection. C. Sexual dimorphism then evolves, causing the sexes to come closer to their respective phenotypic optima, but some antagonism remains. D. Finally, the sexes achieve their optima and the antagonism is completely resolved. Redrawn after information presented in [16]. 


\section{How sex-specific selection affects disease architecture}

241 One implication of sexual antagonism is the maintenance of deleterious genetic variation at higher

242 population frequency than would be expected from mutation-selection balance $[43,44]$. This leads us to

243 consider its role in susceptibility to common, genetically complex disorders. Consistent with this

244 reasoning, mathematical simulation predicts that alleles that are under sex-differential selection (including

245 sexually antagonistic ones) contribute disproportionately to genetic variation underlying disease

246 phenotypes [14]. We now discuss in greater depth how sexual antagonism for standing genetic variation

247 might contribute to the genetic architecture of complex traits.

\section{Unequal endophenotype outcome}

250 For common, complex diseases, risk loci are unlikely to cause disease directly, rather they affect a 251 quantitative trait that is biologically linked to the disease (an endophenotype) and becomes a risk factor 252 when the trait value exceeds a certain threshold [56]. This is derived from the endophenotype hypothesis 253 of psychiatric disorders [57], but can be extended to many disorders, examples of which include the 254 relationship between adiponectin level and Type 2 Diabetes [58], and triglyceride level with coronary 255 artery disease [59].

256 For a correlated trait (with the same genetic architecture between the sexes) an extreme 257 endophenotype value might be beneficial to one sex but detrimental to the other because of other factors, 258 such as sexual dimorphism in physiology, behaviour or environmental exposure. In practice, the extreme 259 trait value might not be beneficial to either sex, but as long as it remains neutral or weakly deleterious, 260 then the causative alleles persist. One example could be for dyslipidaemia that increases risk of 261 myocardial infarction in men but not in women, likely due to the anti-oxidant effects of oestrogen [60].

263 Equal disease risk but with unequal fitness effects

264 Fitness comprises both survival and reproductive components. One might implicitly assume that the 265 reduction in fitness caused by disease is due to disease-related reduction in survival. However, the effects 266 of disease on the second major component of fitness, reproduction, are not always equal between the sexes. One example of this is schizophrenia, where males have a consistently greater reduction in 268 reproductive success than females [61-63]. A second example is for congenital hypothyroidism, 269 associated with loss in fecundity in women but not in men [64]. These examples illustrate how although 270 the genetic architecture of disease may be the same, the fitness effect on each sex as a result of the disease 271 differs.

\section{Sex-specific migration}

274 It has been suggested that the genetic variation for a sexually antagonistic trait may vary between 275 populations [44], and thus immigration results in the introduction of novel, sexually antagonistic alleles 276 into the host population. Sex-biased immigration will cause alleles that are beneficial to that sex (and thus 277 under net positive selection) to be rapidly introduced into a host population, only for the opposite sex to inherit novel deleterious alleles. 
The same principle could be applied to resolved antagonism. For example, methylation is both sex-specific [65,66] and population-specific [67]. It is also proposed as a means by which sexual antagonism can be resolved because it prevents a deleterious allele from being expressed in one sex. The sex-specific migration results in novel combinations of methylated genes increasing the prevalence of extreme (deleterious) phenotypes.

Although obtaining empirical evidence for these processes may be challenging, there is good evidence for large-scale, sex-specific migrations amongst historical human populations from Central Asia [66,68], the Iberian Penninsula [69], the British Isles [70,71], Central Africa [72], Indonesia [73], and globally $[74,75]$. Furthermore, these mechanisms could provide a novel explanation for the outbreeding depression observed in wild animal populations.

\section{Sexually antagonistic pleiotropy}

291 We define sexually antagonistic pleiotropy as the deleterious effect of an allele on a fitness-related 292 phenotype in one sex, with a gain in fitness in the other sex through a different phenotype (Figure 2b-c).

293 One example of this comes from quantitative genetics in which cholesterol levels in men are inversely 294 correlated with height in women [76]. Body height in humans is sexually antagonistic, with high values 295 increasing male fitness but reducing that of females [42]. Thus, selection for shorter females causes a 296 maladaptive response in males by raising their cholesterol levels. Indirect empirical evidence indicates 297 that pleiotropic genes are indeed less able to escape sexual antagonism [47,77], and thus the involvement 298 of pleiotropic genes in disease risk seems likely to be amplified by sex-specific selection. 
For SNP-based association testing, the basic approach to identifying sex-specific effects is to analyse each sex separately, i.e. sex-stratified. In comparison to joint tests, this approach is limited due to the loss in power caused by partitioning of the sample [78]. A common follow-up to the sex-stratified tests is to determine whether the association statistics for each sex are significantly different from one another. Many main-effect studies incorporate sex as a covariate into the analysis, i.e. they are controlling for the effect. However, whilst this approach acknowledges sex-effects it doesn't allow for their detection. For binary traits with a prevalence of less than 1\% inclusion of known covariates actually reduces power [79].

A joint analysis that incorporates a genotype-by-sex interaction term tests the difference in allele frequencies between male and female cases, given their allele frequencies in controls. It is thus more suited to identifying genetic differences in trait architecture between males and females rather than for main effects. The regression model with which to test for genotype-by-sex interactions in an unrelated population sample, is: $\mathrm{Y}[\mathrm{G}, \mathrm{S}]=\beta_{0}+\beta_{\mathrm{G}} \mathrm{G}+\beta_{\mathrm{S}} \mathrm{S}+\beta_{\mathrm{GxS}}(\mathrm{G} \times \mathrm{S})+\varepsilon$, where $\mathrm{Y}$ is the phenotype value, $\mathrm{G}$ is the genotype, $\mathrm{S}$ is the sex, $\beta$ is the standardised regression coefficient of each variable, and $\varepsilon$ is the error [80]. Other covariates, such as those used to correct for population stratification, can also be incorporated into this model. The tests can be performed using open-source software, e.g. PLINK [81] and GenAbel [82], although to the best of our knowledge only one GWAS [83] and two (candidate gene) studies have done so $[84,85]$. For family trio data, a joint, interaction analysis is also possible, exemplified by use of a customdesigned case/pseudo-control test that detected two loci for autism risk [86]. Meta-analysis of GWAS data is a powerful and routine approach to increase power in large heterogeneous sample collections, and an algorithm has been developed in which both sex-specific and main effects can be tested for in a metaanalysis without loss in power [87,88]. An alternative approach (developed for GxE or GxG interactions but applicable to $\mathrm{GxS}$ ) is to use only loci for which the variance differs significantly between genotypes [89]. This reduces the number of tests whilst enriching for loci most likely to have an interaction component.

The statistical behaviour of genotype-by-sex tests must be assumed to be similar to genotypeby-environment tests, in which the interaction term is binary, obligatory and ideally has the same distribution in case and control populations. Power calculations can potentially be undertaken using such software as Gene-Environment iNteraction Simulator (GENS) [90] and GxEscan [91]. Analytical hazards when using an interaction term include artifactual population substructure [92] and incorrect control of confounders [93] other than sex, such as age, ethnicity, or socio-economic background. Interesting opposite effect direction effects may arise $[24,94]$ but are hard to interpret without replication or biological validation.

As more sex-specific analyses of GWAS datasets are performed, it would be informative for authors to present sex-specific values for i) Trait heritability, ii) The phenotypic variance accounted for by significant SNPs, iii) Genomic prediction/Risk profile score. Finally, given the extent of sexually dimorphic interaction networks $[4,95,96]$, pathway enrichment and epistasis testing should be rewarding. 


\section{Conclusions}

341 Despite sharing genetic variation, there are profound biological differences between males and females.

342 This can result in different optima for shared traits, sexual antagonism, and sexual dimorphism. Sex-

343 specific selection on an allele can have important effects on its maintenance within a population,

344 potentially allowing deleterious, disease-associated alleles to persist $[14,43,44]$. This predicts the existence

345 of sex-specific architecture, and indeed the recent analyses of large GWAS data sets has brought about an

346 unprecedented rise in the number of robust sex-specific effects in traits of medical relevance (thirty-four

347 loci for twenty-two traits). In fact, we are aware of only one 'sex-sensitive' GWAS that did not reach

348 genome-wide significance [83]. Sex differences in disease presentation are often stated as the reason for

349 investigating sex-specific genetic effects, but given that sexual dimorphism is a resolution of sexual

350 antagonism, sexually monomorphic traits are more likely to harbour unresolved conflict and thus also

351 have sex-specific genetic architecture.

352 Although we have partially excluded sex hormones from sex differences in genetic architecture,

353 they are a major driver of sex-specific gene expression [4]. Furthermore, as their mode of action is to

354 activate transcription factors, SNPs that alter binding-sites for sex hormone-induced transcription factors

355 will have a sex-dependent effect on gene expression. This mechanism is likely to explain some of the sex-

356 specific GWAS results. Alternatively sex-specific epigenetic modification e.g. methylation [97], will

357 inhibit gene expression, masking any functional variation in one sex but not the other. One example of this

358 is known for the ZPBP2 gene and asthma [98].

359 Much of what is known about sexual antagonism has been obtained through studies on wild and

360 laboratory animal populations, as well as mathematical modelling. Identification of the molecular genetic

361 basis of fitness and of sexual antagonism in model organisms would not only confirm the empirical

362 observations but also provide a grounding for studies of sex-specific genetic architecture in humans.

363 Equally so, ecological studies in humans could also provide interesting perspectives, for example how

364 ecological factors influence selection on specific traits to produce varying degrees of sexually concordant

365 or sexually antagonistic selection across populations [99]. There is evidence from divergent species of

366 weak sex-specific trans-eQTLs [26,100], sex-specific residual genetic architecture [101] and sex-specific

367 epistasis [33]. These studies indicate that many modifier loci for common, complex disorders could be

368 sex-specific. One example of this is age-at-onset of Parkinson's disease being reduced in males only, by

369 the catechol-O-methyltranferase gene Val158 allele [102]. Many monogenic disorders originate from

370 mutations in sex-linked or mitochondrial genes that, because of their transmission dynamics, are under

371 sex-dependent selection. However, the role of common genetic variation on these chromosomes in

372 complex traits is limited by lack of coverage of genotyping chips and suitable analytical methods.

373 We anticipate that analysis of GWAS data with respect to sex, encouraged by both evolutionary

374 genetics and recent results presented in this review, will generate many more significant findings and

375 highlight the potential role of sex-specific and sexually antagonistic selection as a potent force in human

376 genetic architecture. Finally, we hope that the identification of sex-specific genetic aetiologies in what

377 otherwise appears to be the same disease will result in the development of more effective, sex-specific

378 therapies. 


\section{Acknowledgements}

The authors wish to thank laboratory colleagues - Dr Fiona Ingleby, Ms Tanya Pennell, Ms Katrine Lund-

Hansen - for their careful reading of the manuscript and insightful comments.

\section{References}

1. Kondrashov AS (1982) Selection against harmful mutations in large sexual and asexual populations. Genet Res Camb 40: 325332.

2. Hartfield M, Keightley PD (2012) Current hypotheses for the evolution of sex and recombination. Integrative Zoology 7: 192209.

3. Mittelstrass K, Ried JS, Yu Z, Krumsiek J, Gieger C et al. (2011) Discovery of sexual dimorphisms in metabolic and genetic biomarkers. PLoS Genetics 7: e1002215.

4. van Nas A, Guhathakurta D, Wang SS, Yehya N, Horvath S et al. (2009) Elucidating the role of gonadal hormones in sexually dimorphic gene coexpression networks. Endocrinology 150: 1235-1249.

5. Bermejo-Alvarez P, Rizos D, Rath D, Lonergan P, Gutierrez-Adan A (2010) Sex determines the expression level of one third of the actively expressed genes in bovine blastocysts. Proc Nat Acad Sci USA 107: 3394-3399.

6. Sandberg K, Verbalis JG (2013) Sex and the basic scientist: is it time to embrace Title IX? Biol Sex Differ 4: 13.

7. Reich DE, Lander ES (2001) On the allelic spectrum of human disease. Trends Genet 17: 502-510.

8. Pritchard JK, Cox NJ (2002) The allelic architecture of human disease genes: common disease-common variant...or not? Human Molecular Genetics 11: 2417-2423.

9. Manolio TA, Collins FS, Cox NJ, Goldstein DB, Hindorff LA et al. (2009) Finding the missing heritability of complex diseases. Nature 461: 747-753.

10. Lee SH, Harold D, Nyholt DR, AZNGene Consortium, International Endogene Consortium et al. (2013) Estimation and partitioning of polygenic variation captured by common SNPs for Alzheimer's disease, multiple sclerosis and endometriosis. Human Molecular Genetics 22: 832-841.

11. Eichler EE, Flint J, Gibson G, Kong A, Leal SM et al. (2010) Missing heritability and strategies for finding the underlying causes of complex disease. Nature Reviews Genetics 11: 446-450.

12. Ober C, Loisel DA, Gilad Y (2008) Sex-specific genetic architecture of human disease. Nature Reviews Genetics 9: 911-922.

13. Lande R (1980) Sexual dimorphism, sexual selection, and adaptation in polygenic characters. Evolution 34: 292-305.

14. Morrow EH, Connallon T (2013) Implications of sex-specific selection for the genetic basis of disease. Evolutionary Applications. In press.

15. Chippindale AK, Gibson JR, Rice WR (2001) Negative genetic correlation for adult fitness between sexes reveals ontogenetic conflict in Drosophila. Proc Nat Acad Sci USA 98: 1671-1675.

16. Bonduriansky R, Chenoweth SF (2009) Intralocus sexual conflict. Trends Ecol Evol 24: 280-288.

17. Falconer, D. S. and Mackay, T. F. C. (1996) Introduction to quantitative genetics. Harlow: Pearson Education Limited.

18. Pan L, Ober C, Abney M (2007) Heritability estimation of sex-specific effects on human quantitative traits. Genetic Epidemiology 31: 338-347.

19. Bhupathy P, Haines CD, Leinwand LA (2010) Influence of sex hormones and phytoestrogens on heart disease in men and women. Women's Health (Lond Engl) 6: 77-95.

20. Penaloza C, Estevez B, Orlanski S, Sikorska M, Walker R et al. (2009) Sex of the cell dictates its response: differential gene expression and sensitivity to cell death inducing stress in male and female cells. FASEB J 23: 1869-1879.

21. Arnold AP, Chen X (2009) What does the "four core genotypes" mouse model tell us about sex differences in the brain and other tissues? Front Neuroendocrinol 30: 1-9.

22. Ngun TC, Ghahramani N, Sánchez FJ, Bocklandt S, Vilain E (2011) The genetics of sex differences in brain and behavior. Front Neuroendocrinol 32: 227-246.

23. Dimas AS, Nica AC, Montgomery SB, Stranger BE, Raj T et al. (2012) Sex-biased genetic effects on gene regulation in humans. Genome Research. In press.

24. Kong A, Thorleifsson G, Stefansson H, Masson G, Helgason A et al. (2008) Sequence variants in the RNF212 gene associate with genome-wide recombination rate. Science 319: 1398-1401.

25. Massouras A, Waszak SM, Albarca-Aguilera M, Hens K, Holcombe W et al. (2012) Genomic variation and its impact on gene expression in Drosophila melanogaster. PLoS Genetics 8: e1003055.

26. Bhasin JM, Chakrabarti E, Peng D-Q, Kulkarni A, Chen X et al. (2008) Sex specific gene regulation and expression QTLs in mouse macrophages from a strain intercross. PLoS One 3: e1435.

27. Lehtovaara A, Schielzeth H, Flis I, Friberg U (2013) Heritability of life span is largely sex limited in Drosophila. Am Nat 182: 653-665.

28. Harbison ST, Sehgal A (2008) Quantitative genetic analysis of sleep in Drosophila melanogaster. Genetics 178: 2341-2360.

29. wang S, Yehya N, Schadt EE, Wang H, Drake TA et al. (2006) Genetic and genomic analysis of a fat mass trait with complex inheritance reveals marked sex specificity. PLoS Genetics 2: e15.

30. Kenney-Hunt JP, Wang B, Norgard EA, Fawcett G, Falk D et al. (2008) Pleiotropic patterns of quantitative trait loci for 70 murine skeletal traits. Genetics 178: 2275-2288.

31. dePaula FJ, Dick-de-Paula I, Bornstein S, Rostama B, Le P et al. (2011) VDR haploinsufficiency impacts body composition and skeletal acquisition in a gender-specific manner. Cacif Tissue Int 89: 179-191.

32. Waskar M, Landis GN, Shen J, Curtis C, Tozer K et al. (2009) Drosophila melanogaster p53 has developmental stage-specific and sex-specific effects on adult life span indicative of sexual antagonistic pleiotropy. Aging (Albany NY) 1:903-936.

33. Magwire MM, Yamamoto A, Carbone MA, Roshina NV, Symonenko AV et al. (2010) Quantitative and molecular genetic analyses of mutations increasing Drosophila life span. PLoS Genetics 6: e1001037.

34. van Doorn GS (2009) Intralocus sexual conflict. Ann N Y Acad Sci 1168: 52-71. 
35. Parker GA (1982) Why are there so many tiny sperm? Sperm competition and the maintenance of two sexes. Journal of Theoretical Biology 96: 281-294.

36. Schärer L, Rowe L, Arnqvist G (2012) Anisogamy, chance and the evolution of sex roles. Trends Ecol Evol 27: 260-264.

37. Kokko H, Jennions MD (2008) Parental investment, sexual selection and sex ratios. J Evol Biol 21: 919-948.

38. Cox RM, Calsbeek R (2009) Sexually antagonistic selection, sexual dimorphism, and the resolution of intralocus sexual conflict. Am Nat 173: 176-187.

39. Abbott JK, Morrow EH (2011) Obtaining snapshots of genetic variation using hemiclonal analysis. Trends Ecol Evol 26: 359368.

40. Brommer JE, Kirkpatrick M, Qvarnström A, Gustafsson L (2007) The intersexual genetic correlation for lifetime fitness in the wild and its implications for sexual selection. PLoS One 8: e744.

41. Foerster K, Coulson T, Sheldon BC, Pemberton JM, Clutton-Brock TH et al. (2007) Sexually antagonistic genetic variation for fitness in red deer. Nature 447: 1107-1110.

42. Stulp G, Kuijper B, Buunk AP, Pollet TV, Verhulst S (2012) Intralocus sexual conflict over human height. Biol Lett 8: 976-978.

43. Dean R, Perry JC, Pizzari T, Mank JE, Wigby S (2012) Experimental evolution of a novel sexually antagonistic allele. PLoS Genetics 8: e1002917.

44. Connallon T, Clark AG (2012) A general population genetic framework for antagonistic selection that accounts for demography and recurrent mutation. Genetics 190: 1477-1489.

45. Rice WR (1992) Sexually antagonistic genes: experimental evidence. Science 256: 1436-1439.

46. Prasad NG, Bedhomme S, Day T, Chippindale AK (2007) An evolutionary cost of separate genders revealed by male-limited expression. Am Nat 169: 29-37.

47. Innocenti P, Morrow EH (2010) The sexually antagonistic genes of Drosophila melanogaster. PLoS Biol 8: e1000335.

48. Harano T, Okada K, Nakayama S, Miyatake T, Hosken DJ (2010) Intralocus sexual conflict unresolved by sex-limited trait expression. Current Biology 20: 2036-2039.

49. Gallach M, Betrán E (2011) Intralocus sexual conflict resolved through gene duplication. Trends Ecol Evol 26: 222-228.

50. Assis R, Bachtrog D (2013) Neofuntionalization of young duplicate genes in Drosophila. Proc Nat Acad Sci USA 110: 1740917414.

51. Ellegren H, Parsch J (2007) The evolution of sex-biased genes and sex-biased gene expression. Nature Reviews Genetics 8: 689698.

52. Hosken DJ (2011) Gene duplication might not resolve intralocus sexual conflict. Trends Ecol Evol 26: 556-557.

53. Mank JE (2009) Sex chromosomes and the evolution of sexual dimorphism: lessons from the genome. Am Nat 173: 141-150.

54. Pischedda A, Chippindale AK (2006) Intralocus sexual conflict diminishes the benefits of sexual selection. PLoS Biol 4: 20992113.

55. Pennell TM, Morrow EH (2013) Two sexes, one genome: the evolutionary dynamics of intralocus sexual conflict. Ecology and Evolution. In press.

56. Li Y, Huang J, Amos CI (2012) Genetic association analysis of complex diseases incorporating intermediate phenotype information. PLoS One 7: e46612.

57. Gottesman II, Gould TD (2003) The endophenotype concept in psychiatry: etymology and strategic intentions. Am J Psychiatry 160: 636-645.

58. Dastani Z, Hivert MF, Timpson N, Perry JR, Yuan X et al. (2012) Novel loci for adiponectin levels and their influence on type 2 diabetes and metabolic traits: a multi-ethnic meta-analysis of 45,891 individuals. PLoS Genetics 8: e1002607.

59. Do R, Willer CJ, Schmidt EM, Sengupta S, Gao C et al. (2013) Common variants associated with plasma triglycerides and risk for coronary artery disease. Nat Genet $45: 1345-1352$.

60. Madssen E, Laugsand LE, Wiseth R, Mørkedal B, Platou C et al. (2013) Risk of acute myocardial infarction: dyslipidemia more detrimental for men than women. Epidemiology 24: 637-642.

61. Haukka J, Suvisaari J, Lönnqvist J (2003) Fertility of patients with schizophrenia, their siblings, and the general population: a cohort study from 1950 to 1959 in Finland. Am J Psychiatry 160: 460-463.

62. Power RA, Kyaga S, Uher R, MacCabe JH, Långström N et al. (2013) Fecundity of patients with schizophrenia, autism, bipolar disorder, depression, anorexia nervosa, or substance abuse vs their unaffected siblings. JAMA Psychiatry 70: 22-30.

63. Svensson AC, Lichtenstein P, Sandin S, Hultman CM (2007) Fertility of first-degree relatives of patients with schizophrenia: a three generation perspective. Schizophr Res 91: 238-245.

64. Hassani Y, Larroque B, Dos Santos S, Ecosse E, Bouyer J et al. (2012) Fecundity in young adults treated early for congenital hypothyroidism is related to the initial severity of the disease: a longitudinal population-based cohort study. J Clin Endocrinol Metab 97: 1897-1904.

65. Scarborough K, Hattman S, Nur U (1984) Relationship of DNA methylation level to the presence of heterochromatin in mealybugs. Mol Cell Biol 4: 599-603.

66. Xu H, Wang F, Liu Y, Yu Y, Gelernter J et al. (2013) Sex-biased methylome and transcriptome in human prefrontal cortex. Human Molecular Genetics. In press.

67. Fraser HB, Lam LL, Neumann SM, Kobor MS (2012) Population-specificity of human DNA methylation. Genome Biology 13: R8.

68. Pérez-Lezaun A, Calafell F, Comas D, Mateu E, Bosch E et al. (1999) Sex-specific migration patterns in Central Asian populations, revealed by analysis of Y-chromosome short tandem repeats and mtDNA. Am J Hum Genet 65: 208-219.

69. Hurles ME, Veitia R, Arroyo E, Armenteros M, Betranpetit J et al. (1999) Recent male-mediated gene flow over a linguistic barrier in Iberia, suggested by analysis of a Y-chromosomal DNA polymorphism. Am J Hum Genet 65: 1437-1448.

70. Capelli C, Redhead N, Abernethy JK, Gratrix F, Wilson JF et al. (2003) A Y chromosome census of the British Isles. Current Biology 13: 979-984.

71. Wilson JF, Weiss DA, Richards M, Thomas MG, Bradman N et al. (2001) Genetic evidence for different male and female roles during cultural transitions in the British Isles. Proc Nat Acad Sci USA 98: 5078-5083.

72. Verdu P, Becker NS, Froment A, Georges M, Grugni V et al. (2013) Sociocultural behavior, sex-biased admixture, and effective population sizes in Central African Pygmies and non-Pygmies. Mol Biol Evol 30: 918-937.

73. Tumonggor MK, Karafet TM, Hallmark B, Lansing JS, Sudoyo H et al. (2013) The Indonesian archipelago: an ancient genetic highway linking Asia and the Pacific. J Hum Genet 58: 165-173.

74. Wilder JA, Kingan SB, Mobasher Z, Pilkington MM, Hammer MF (2004) Global patterns of human mitochondrial DNA and Ychromosome structure are not influenced by higher migration rates of females versus males. Nat Genet 36: 1122-1125.

75. Heyer E, Chaix R, Pavard S, Austerlitz F (2012) Sex-specific demographic behaviours that shape human genomic variation. Mol Ecol 21: 597-612. 
76. Stearns SC, Govindaraju DR, Ewbank D, Byars SG (2012) Constraints on the coevolution of contemporary human males and females. Proc R Soc Lond B Biol Sci 279: 4836-4844.

77. Mank JE, Hultin-Rosenberg L, Zwahlen M, Ellegren H (2008) Pleiotropic constraint hampers the resolution of sexual antagonism in vertebrate gene expression. Am Nat 171:35-43.

78. Behrens G, Winkler TW, Gorski M, Leitzmann MF, Heid IM (2011) To stratify or not to stratify: power considerations for population-based genome-wide association studies of quantitative traits. Genetic Epidemiology 35: 867-879.

79. Pirinen M, Donnelly P, Spencer CC (2012) Including known covariates can reduce power to detect genetic effects in casecontrol studies. Nat Genet 44: 848-851.

80. Lynch, M. and Walsh, B. (1998) Genetics and analysis of quantitative traits. Sunderland, MA: Sinauer Associates, Inc.

81. Purcell S, Neale B, Todd-Brown K, Thomas L, Ferreira MA et al. (2007) PLINK: a tool set for whole-genome association and population-based linkage analyses. Am J Hum Genet 81: 559-575.

82. Aluchenko YS, Ripke S, Isaacs A, van Duijn CM (2007) GenABEL: an R library for genome-wide association analysis. Bioinformatics 23: 1294-1296.

83. Liu CT, Estrada K, Yerges-Armstrong LM, Amin N, Evangelou E et al. (2012) Assessment of gene-by-sex interaction effect on bone mineral density. J Bone Miner Res 27: 2051-2064.

84. Loisel DA, Tan Z, Tisler CJ, Evans MD, Gangnon RE et al. (2011) IFNG genotype and sex interact to influence the risk of childhood asthma. J Allergy Clin Immunol 128: 524-531.

85. McCarthy JJ, Somji A, Weiss LA, Steffy B, Vega R et al. (2009) Polymorphisms of the scavenger receptor class B member 1 are associated with insulin resistance with evidence of gene by sex interaction. J Clin Endocrinol Metab 94: 1789-1796.

86. Lu AT, Cantor RM (2012) Allowing for sex differences increases power in a GWAS of multiplex Autism families. Molecular Psychiatry 17: 215-222.

87. Magi R, Lindgren CM, Morris AP (2010) Meta-analysis of sex-specific genome-wide association studies. Genetic Epidemiology 34: 846-853.

88. Strawbridge RJ, Dupuis J, Prokopenko I, Barker A, Ahlqvist E et al. (2011) Genome-wide association identifies nine common variants associated with fasting proinsulin levels and provides new insights into the pathophysiology of type 2 diabetes. Diabetes 60: 2624-2634.

89. Paré G, Cook NR, Ridker PM, Chasman DI (2010) On the use of variance per genotype as a tool to identify quatitative trait interaction effects: a report from the Women's Genome Health Study. PLoS Genetics 6: e1000981.

90. Amato R, Pinelli M, D'Andrea D, Miele G, Nicodemi M et al. (2010) A novel approach to simulate gene-environment interactions in complex diseases. BMC Bioinformatics 11:8.

91. Gauderman WJ, Zhang P, Morrison JL, Lewinger JP (2013) Finding novel genes by testing G $\times \mathrm{E}$ interactions in a genome-wide association study. Genetic Epidemiology 37: 603-613.

92. Voorman A, Lumley T, McKnight B, Rice K (2011) Behavior of QQ-plots and genomic control in studies of gene-environment interaction. PLoS One 6: e19416.

93. Keller MC (2013) Gene $\times$ Environment interaction studies have not properly controlled for potential confounders: the problem and the (simple) solution. Biol Psychiatry. In press.

94. Radulescu E, Sambataro F, Mattay VS, Callicott JH, Straub RE et al. (2012) Effect of schizophrenia risk-associated alleles in SREB2 (GPR85) on functional MRI phenotypes in healthy volunteers. Neuropsychopharmacology 38: 341-349.

95. Hansen MEB, Kulathinal RJ (2013) Sex-biased networks and nodes of sexually antagonistic conflict in Drosophila. International Journal of Evolutionary Biology 2013: 545392.

96. McCoy CM, Nicholas DQ, Masters KS (2012) Sex-related differences in gene expression by porcine aortic valvular interstitial cells. PLoS One 7: e39980.

97. Kobayashi H, Sakurai T, Miura F, Imai M, Mochiduki K et al. (2013) High-resolution DNA methylome analysis of primordial germ cells identifies gender-specific reprogramming in mice. Genome Research 23: 616-627.

98. Naumova AK, Al Tuwaijri A, Morin A, Vaillancourt VT, Madore AM et al. (2013) Sex- and age-dependent DNA methylation at the 17q12-q21 locus associated with childhood asthma. Human Genetics 132: 811-822.

99. Innocenti P, Morrow EH (2010) A joint index for the intensity of sex-specific selection. Evolution 64: 2775-2778.

100. Derome N, Bougas B, Rogers SM, Whiteley AR, Labbe A et al. (2008) Pervasive sex-linked effects on transcription regulation as revealed by expression quantitative trait loci mapping in lake whitefish species pairs (Coregonus sp., Salmonidae). Genetics 179: 1903-1917.

101. Perry GML, Nehrke KW, Bushinsky DA, Reid R, Lewandowski KL et al. (2012) Sex modifies genetic effects on residual variance in urinary calcium excretion in rat (Rattus norvegicus). Genetics 191: 1003-1013.

102. Klebe S, Golmard J-L, Nalls MA, Saad M, Singleton AB et al. (2013) The Val158Met COMT polymorphism is a modifier of the age at onset in Parkinson's disease with a sexual dimorphism. J Neurol Neurosurg Psychiatry 84: 666-673. 